Towards logistics systems parameter optimisation through the use of response surfaces

Peer-reviewed author version

RAMAEKERS, Katrien; JANSSENS, Gerrit K. \& Van Landeghem, H. (2006) Towards logistics systems parameter optimisation through the use of response surfaces. In: 4OR: A Quarterly Journal of Operations Research, 4(4). p. 331-342.

DOI: $10.1007 / \mathrm{s} 10288-006-0024-2$

Handle: http://hdl.handle.net/1942/1518 


\title{
Towards logistics systems parameter optimisation through the use of response surfaces
}

\author{
Katrien Ramaekers • Gerrit K. Janssens • \\ Hendrik Van Landeghem
}

\begin{abstract}
Logistics systems have to cope with uncertainties in demand, in lead times, in transport times, in availability of resources and in quality. Management decisions have to take these uncertainties into consideration. An evaluation of decisions may be done by means of simulation. However, not all stochastic phenomena are of equal importance. By design of simulation experiments and making use of response surfaces, the most important phenomena are detected and their influence on performance estimated. Once the influence of the phenomena is known, this knowledge may be used to determine the optimal values of some decision parameters. An illustration is given on how to use response surfaces in a real-world case. A model is built in a logistics modelling software. The decision parameters have to be optimised for a specific objective function. Experiments are run to estimate the response surface. The validity of the response surface with few observations is also tested.
\end{abstract}

Keywords Simulation-Optimisation - Response surfaces · Experimental design · Regression · Logistics

K. Ramaekers $(\varangle) \cdot$ G. K. Janssens

Operations Management and Logistics, Hasselt University,

Agoralaan - Building D, 3590 Diepenbeek, Belgium

e-mail: katrien.ramaekers@uhasselt.be

G. K. Janssens

e-mail: gerrit.janssens@uhasselt.be

H. Van Landeghem

Industrial Management, Ghent University, Technologiepark 903,

9052 Zwijnaarde (Gent), Belgium

e-mail: hendrik.vanlandeghem@UGent.be 


\section{Introduction}

The design of logistics networks requires decisions on the strategic, tactical and operational levels. Operations Research has been focusing on specific topics mostly regarding the operational aspects as inventory levels or vehicle routing. But today the integration of logistics, over the boundaries of companies, is at stake. Supply chains are designed in order to let the flow of goods, finance and information be organised in a smooth way. Potential savings can be generated by the integration of the design of strategic supply chain networks with the determination of tactical production-distribution allocations.

In the literature, several models and their corresponding solution algorithms for the design of global logistics systems are described. Goetschalckx et al. (2002) give an overview of the application of mathematical programming models in the strategic design and improvement of global logistics systems. Vidal and Goetschalckx (1997) identify several lacking features and opportunities for research in the methodology for the strategic and tactical design of global logistics systems. Goetschalckx et al. (2002) summarize the international characteristics of published strategic global logistics models. Among the international characteristics of these global logistics systems, stochastic features make up an important set. Hodder and Dincer (1986) state that there is a vast literature on domestic location problems, which are of deterministic nature but when international location problems are considered, uncertainty can be a very important issue. However, few published models take into account the stochastic features because the incorporation of stochastic elements into the mathematical programming models for supply chain design yields very difficult formulations. Vidal and Goetschalckx (1996) identify the ability to include key stochastic features in the formulations and analyze them in a more efficient way as one of the most fascinating challenges of future mathematical global logistics models.

The organisation of an international logistics network requires the tuning of many parameters in order to run it efficiently and stochastic features have to be taken into account. Classical optimisation methods may fail because of the complexity of the problem. Therefore simulation models are used to model the networks: its components, its random characteristics and its parameters. But the outcome of a simulation is random, and when it comes to optimisation one is confronted with an additional problem of uncertainty of replication. Simulation and optimisation were often seen as two separate disciplines in the area of operations research. However, in recent years, the combination of simulation and optimisation has developed steadily and has increased in popularity. Simulation optimisation is the practice of linking an optimisation method with a simulation model to determine appropriate settings of certain input parameters so as to maximize the performance of the simulated system.

Techniques for simulation optimisation vary greatly depending on the exact problem setting. Four major categories of simulation optimisation methods 
can be distinguished: design of experiments, guided search methods, indirect optimisation and statistical methods. A first class of simulation optimisation methods are Design of Experiments techniques (Vanmaele and Van Landeghem 1995). These techniques provide a way to set up the complete experimental design before the experimentation process begins. Design of experiment methods can in general only be applied to discrete variables. Several schemes for setting up experimental designs are known from the literature. Some examples are one factor at a time, full factorial experimental design and the Taguchi method (Ross 1988).

Guided search methods are a second class of simulation optimisation methods. In guided search methods, the result of the previous experiment is used to decide on the factor values that will be changed to run the following experiment. Three classes of guided search methods are distinguished. Numerical methods like the Hooke and Jeeves method (Hooke and Jeeves 1961) are based on the idea that if a direction has produced a favourable change in the optimal value, then one should continue to move in this direction (Jacobson and Schruben 1989). Gradient based methods are based on the calculation of gradients in order to move through the search space. Several techniques can be used to estimate the response gradient: finite differences, likelihood ratios, perturbation analysis and frequency domain experimentation (Andradottir 1995, 1996). Random search methods include metaheuristics such as tabu search, genetic algorithms and simulated annealing. Although these methods are generally designed for combinatorial optimisation in the deterministic context, they have been quite successful when applied to simulation optimisation (Olafsson and Kim 2002). Paul and Chanev (1998) demonstrate the capability of genetic algorithms to solve problems in the area of complex simulation model optimisation. Haddock and Mittenthal (1992) investigate the feasibility of using a simulated annealing algorithm in conjunction with a simulation model to find the optimal parameter levels at which to operate a system. Dengiz and Alabas (2000) use a tabu search algorithm in conjunction with a simulation model to find the optimal parameter levels.

A third class are the statistical methods (Goldsman et al. 2002; Ho et al. 2000; Pichitlamken and Nelson 2001). These methods are mostly used when the optimisation process involves selecting the best of a finite number of alternatives and the parameters are discrete. Some examples of statistical methods are ranking and selection, selection with memory and multiple comparison procedures.

The fourth class of simulation optimisation methods, indirect optimisation or response surface methodology (RSM), is useful when input factors are quantitative and continuous. A response surface is a meta-model, i.e., it is a regression model which models the output results of a simulation model (Myers et al. 1989).

Response surfaces are a meta-model, i.e., they make up a regression model of the response of the simulation model to be explained by parameters to be set during simulation experiments. Response surface methodology (RSM) studies the local geography of the response surface through the response function. 
Through this function in the neighbourhood of the optimal value, it is able to determine this optimal value. It is especially useful where a response of interest is influenced by multiple variables. RSM is a very general methodology that uses well-known and well-studied statistical tools. In this paper, an illustration is given on how to use response surfaces in a real-world case. We make use of a simulation model in order to generate a performance measure for a logistics network and explain its variance by a set of parameter values. This approach is useful in order to identify important parameters and their influence, in order to assign their optimal values in terms of a certain objective.

\section{Illustrative example: the background story}

To illustrate the use of response surfaces in logistics systems parameter optimisation, a model has been developed in a software called Logistics Redesign, a dynamic simulator that provides solutions to supply chain planning. 'Prima', the company being modelled, produces personal computers at a plant in Riga, Latvia. Computers are delivered to a distribution centre (DC) in Lübeck by sea, and then further to its customers. The company's customers (computer retailers) are located in France, Belgium, Germany and Italy. The company has 13 customers, each with a random demand, which are assumed to follow a normal distribution. Computers are transported from the plant to the distribution centre by ship and from the distribution centre to the customers by truck. The speed of both the road and the sea service is also a random variable, which are assumed to follow a normal distribution.

The case study is based on hypothetical data. They do not represent reallife data from a company. Business in the East-European new members of the European Union are currently investigating a way to penetrate markets in the Western part of Europe. From a business viewpoint a move of manufacturing facilities is observed towards the East of Europe, but this leads to a need for the design of a supply chain from East to West both in manufactured products as in service after sales.

The objective of the company is to minimize the company's logistics costs, represented by the variable $Y$. The logistic costs are composed of inventory costs, warehouse costs, administration costs, customer service costs and transportation costs. All costs are expressed in Euro. The transportation costs for the road service include a fixed cost per trip, a variable cost related to the weight $w$ (in tons), a variable cost related to the distance $d$ (in $\mathrm{km}$ ) travelled and a variable cost related to the time $t$ (in hours) travelled. In the example the road transportation cost is calculated as: $100+100 w+0.1 d+10 t$. The transportation costs for the sea service include a fixed cost per trip, a variable cost related to the weight $w$ (in tons), and a variable cost related to the time $t$ (in hours) travelled. The sea transportation cost is calculated in the example as: $50+20 w+15 t$. The yearly inventory costs are $25 \%$ of the product value. A reactive inventory control system with a reorder point and fixed order quantity is used. The administration and customer service costs consist of fixed costs for 
each order and for the dispatching of a consignment. The optimum level of the following six factors has to be determined: the reorder point of the DC, the fixed order quantity of the DC, the average speed and daily capacity of the road service, and the average speed and daily capacity of the sea service.

The logistics system under study addresses the impact of operational decisions or controllable factors (i.e., reorder point, fixed order quantity, capacity of the road service and capacity of the sea service) and system parameters or uncontrollable factors (i.e., speed of the road service and speed of the sea service).

In order to leave open as many options as possible, we do not impose any constraints on the reorder point, the fixed order quantity and the capacities of both the road and sea services. However, the average speed of the road service is limited to $80 \mathrm{~km} / \mathrm{h}$ and the average speed of the sea service is limited to $36 \mathrm{~km} / \mathrm{h}$.

The simulation model is built in Logistics Redesign, a dynamic simulator that provides solutions to supply chain planning. Each simulation run simulated 365 days and computation time per run is around $2 \mathrm{~min}$. The same initial parameter settings are used for each simulation run. Five replications of the simulation run are made for each experimental point. The experiments are described in detail further on.

\section{A two-stage procedure explained using the illustrative example}

Phase 1: First-order analysis

\section{Step 1: Range determination of each factor}

The first step in determining the optimal values of the logistics systems parameters is to specify an initial region in which to start simulation experiments. At first a central point in the six-dimensional space is defined. The central values of the six factors are chosen at: 125 units for the reorder point, 600 units for the fixed order quantity, $50 \mathrm{~km} / \mathrm{h}$ for the mean speed of the road service, 100 units for the capacity of the road service, $28.83 \mathrm{~km} / \mathrm{h}$ for the mean speed of the sea service, and 450 units for the capacity of the sea service. The lower and upper values for each factor can be found in Table 1 .

Table 1 Levels for the six experimental factors

\begin{tabular}{lrr}
\hline Factor name & Lower & Upper \\
\hline Reorder point $\left(X_{1}\right)$ & 100.00 & 150.00 \\
Fixed order quantity $\left(X_{2}\right)$ & 500.00 & 700.00 \\
Speed road service $\left(X_{3}\right)$ & 40.00 & 60.00 \\
Capacity road service $\left(X_{4}\right)$ & 75.00 & 125.00 \\
Speed sea service $\left(X_{5}\right)$ & 24.71 & 32.94 \\
Capacity sea service $\left(X_{6}\right)$ & 400.00 & 500.00 \\
\hline
\end{tabular}


The experimental points are standardized using the central point value and the range in order to define a new set of variables $x_{i}(i=1, \ldots, 6)$, which have values in the interval $[-1,+1]$ :

$$
x_{1}=\frac{X_{1}-125}{25} ; \quad x_{2}=\frac{X_{2}-600}{100} ; \ldots
$$

This is done to simplify the calculations in further steps.

Step 3: Data collection through experiment runs

Around the central values an experimental design is made up to specify the combinations of the levels of the six factors that need to be evaluated. As a starting point, a first order design is used. A very widely used first order design is the factorial design. In a factorial design, each factor is evaluated at two levels. For this example the level values are shown in Table 1. With six factors, in the full factorial design $2^{6}$ experimental points need to be investigated. The central point is added to the 64 experimental points. For each experimental point, five replications of the simulation are run.

Step 4: First-order model fitting and adequacy check

By using regression analysis, a first order polynomial is fit to the specified points and their simulated results, represented in the variable $Y$. The following first order polynomial was obtained:

$$
Y=573815-1137 x_{1}-4505 x_{2}-31969 x_{3}-13182 x_{4}-11151 x_{5}-14035 x_{6}
$$

The coefficient of determination $R^{2}$ of the regression equals 0.877 . A value for the $F$-test, with degrees of freedom 6 and 318, of 386.771 indicates a significance on the $95 \%$ confidence level of the overall regression. In order to investigate the significance of the individual parameters $x_{i}(i=1, \ldots, 6)$, their $t$ test values, with 318 degrees of freedom are shown in the next vector: $(-1.397,-5.533,-39.26,-16.189,-13.695,-17.236)$. The $t$ test value of the intercept equals 710.157 .

Regarding the individual influence only the influence of factor $x_{1}$ can be discarded (on a 95\% confidence level), under the assumption that the model is a valid model of the real world.

Step 5: Validation of the first-order model

Validation is the "substantiation that a model within its domain of applicability possesses a satisfactory range of accuracy consistent with the intended 
application of the model" (Kleijnen and Sargent 2000). In the literature, two ways of validating a regression model are described: new data collection and data splitting or cross validation (Batmaz and Tunali 2003). We use the first technique to check the validation of the response surface. The simulation is run for a number of randomly chosen combinations of the factors. The output of the runs is compared with the values $Y$, computed from the response surface regression. No significant differences were detected between both sets of values and, by this, the response surface is considered valid.

\section{Step 6: Reduction of the experimental design towards the second stage}

Step 6 is of academic nature in this study. A full factorial design, combined with many replications, may lead to a considerable computation effort. Either from an a priori knowledge or from a pragmatic point of view, it may be useful to discard a number of experimental design points in a rigorous way. By doing this the main effects and the interaction effects, which are assumed to be of importance, still can be computed, while other high-level interactions are considered non-existent. For academic purposes, some experiments were run changing the incorporation of the central point, the number of replications, and the application of a fractional design. This fractional factorial design is chosen to have a resolution IV, which means no main effect is aliased with either main effects or two-factor interactions (Box and Draper 1987). In Table 2, an overview of the designs is given. Table 3 contains a comparison of these designs. A number printed in italic in Table 3 indicates that this parameter (column) is not significant on the $95 \%$ confidence level for the specific design (row). A column with heading $\beta_{i}(i=0, \ldots, 6)$ shows the regression coefficient of the coded variable $x_{i}$.

Because the sixth design gives very good results, compared to the level of simulation effort, this design will be used in the remainder of this paper. Since the first factor is not significant on the $95 \%$ confidence level, the value of the reorder point will be fixed at its central point, 125 units, in the calculations that follow.

Table 2 Overview of designs in the reduction investigation experiment

\begin{tabular}{llllllr}
\hline Design-id & Design type & $\begin{array}{l}\text { Centre } \\
\text { point used }\end{array}$ & $\begin{array}{l}\text { Number } \\
\text { of points }\end{array}$ & Runs/point & $R_{\text {adj }}^{2}$ & $\begin{array}{r}F \text {-test } \\
\text { value }\end{array}$ \\
\hline 1 & Full fact. design & Yes & 65 & 5 & 0.877 & 386.8 \\
2 & Full fact. design & No & 64 & 5 & 0.878 & 383.3 \\
3 & Full fact. design & Yes & 65 & 1 & 0.867 & 70.5 \\
4 & Full fact. design & No & 64 & 1 & 0.867 & 69.5 \\
5 & Fract. fact. design & No & 16 & 1 & 0.801 & 11.1 \\
6 & Fract. fact. design & No & 16 & 5 & 0.858 & 80.3 \\
7 & Fract. fact. design & No & 16 & 20 & 0.843 & 286.2 \\
\hline
\end{tabular}


After fitting a first order polynomial to the simulation results obtained from the experiment, a direction needs to be determined that reduces the logistics costs (response variable $Y$ ). Once determined, further points in this direction are simulated and evaluated until no further logistics costs reduction can be found.

This procedure is called the method of steepest descent (Safizadeh and Thornton 1984; Khuri and Cornell 1988; Box and Draper 1987). Table 4 shows the required data for the steepest descent procedure. The 'base level' row shows the central point values for the five remaining significant variables $\left(X_{2}, X_{3}, X_{4}, X_{5}\right.$ and $\left.X_{6}\right)$. As each variable $X_{i}$ is coded into the interval $[-1,1]$ to a standardised variable using a standardising unit, these values are shown in the unit row. The 'estimated slopes' row shows the improvement per unit of an independent variable. The values correspond to the regression coefficients in Table 3 with design-id equal to 6 . Negative regression coefficients correspond to an improvement in the objective function. The path of steepest descent corresponds to the direction in which the response variable decreases most.

The estimated direction of steepest descent follows the vector of coefficient values of the five significant variables $(-4,410,-32,032,-13,708,-11,642$, $-14,342)$. The length of this vector is $\left[(-4,410)^{2}+(-32,032)^{2}+(-13,708)^{2}+\right.$ $\left.(-11,642)^{2}+(-14,342)^{2}\right]^{1 / 2}=39,682$. A vector of unit length in the direction of steepest descent has coordinates $\triangle x_{2}=-4,410 / 39,682, \triangle x_{3}=-32,032 / 39,682$, $\triangle x_{4}=-13,708 / 39,682, \triangle x_{5}=-11,642 / 39,682$ and $\triangle x_{6}=-14,342 / 39,682$. The coded $\triangle x_{i}$ are converted into the natural variables and their values are shown in the 'vector of unit length'-row.

Table 3 Regression coefficients for the designs identified in Table 2

\begin{tabular}{llllllll}
\hline Design-id & $\beta_{0}$ & $\beta_{1}$ & $\beta_{2}$ & $\beta_{3}$ & $\beta_{4}$ & $\beta_{5}$ & $\beta_{6}$ \\
\hline 1 & 573,815 & $-1,137$ & $-4,506$ & $-31,970$ & $-13,813$ & $-11,152$ & $-14,035$ \\
2 & 573,960 & $-1,137$ & $-4,506$ & $-31,970$ & $-13,813$ & $-11,152$ & $-14,035$ \\
3 & 573,645 & -634 & $-4,788$ & $-32,372$ & $-13,285$ & $-11,023$ & $-14,261$ \\
4 & 573,645 & -634 & $-4,788$ & $-32,372$ & $-13,285$ & $-11,023$ & $-14,261$ \\
5 & 572,440 & -591 & $-4,179$ & $-32,493$ & $-14,140$ & $-11,220$ & $-14,359$ \\
6 & 573,266 & $-1,057$ & $-4,410$ & $-32,032$ & $-13,708$ & $-11,642$ & $-14,342$ \\
7 & 573,433 & -588 & $-4,023$ & $-31,783$ & $-13,635$ & $-11,017$ & $-14,333$ \\
\hline
\end{tabular}

Table 4 Calculation of steepest descent path

\begin{tabular}{llllll}
\hline & $X_{2}$ & $X_{3}$ & $X_{4}$ & $X_{5}$ & $X_{6}$ \\
\hline Base level & 600 & 50 & 100 & 28.83 & 450 \\
Unit & 100 & 10 & 25 & 4.11 & 50 \\
Estimated slopes & 4,410 & 32,032 & 13,708 & 11,642 & 14,342 \\
Vector of unit length & 11.1 & 8.07 & 8.625 & 1.204 & 18.05 \\
\hline
\end{tabular}


Table 5 Subsequent trials on the path of steepest descent

\begin{tabular}{lllllll}
\hline Trial & $X_{2}$ & $X_{3}$ & $X_{4}$ & $X_{5}$ & $X_{6}$ & $\mathrm{Y}$ \\
\hline 1 & 600 & 50 & 100 & 28.83 & 450 & 562,945 \\
2 & 611.1 & 58.07 & 108.625 & 30.034 & 468.05 & 534,070 \\
3 & 622.2 & 66.14 & 117.25 & 31.238 & 486.1 & 518,768 \\
4 & 633.3 & 74.21 & 125.875 & 32.442 & 504.15 & 499,779 \\
5 & 641.3 & 80 & 132.1 & 33.31 & 517.15 & 490,756 \\
6 & 652.4 & 80 & 140.725 & 34.514 & 535.2 & 487,259 \\
7 & 663.5 & 80 & 149.35 & 35.718 & 553.25 & 483,300 \\
8 & 666.1 & 80 & 151.35 & 36 & 557.45 & 483,256 \\
9 & 677.2 & 80 & 159.975 & 36 & 575.5 & 481,542 \\
10 & 688.3 & 80 & 168.6 & 36 & 593.55 & 480,155 \\
11 & 699.4 & 80 & 177.225 & 36 & 611.6 & 481,604 \\
\hline
\end{tabular}

Table 5 shows the parameters of the exploratory runs and the associated cost $Y$ of this procedure for the example. Because the objective is cost minimization, the negative signs in the figures of the estimated slopes in Table 4 are omitted.

At the fifth trial point of the steepest descent path, the constraint on the mean speed of the road service will be violated. Therefore, the point where the path of the steepest descent meets the constraint is determined. This point, $(641.3,80$, $132.1,33.31,517.15)$, will be the next trial point on the path of steepest descent. The modified direction of steepest descent changes into (11.1, 0, 8.625, 1.204, 18.05).

At the eighth trial point the constraint on the mean speed of the sea service will be violated. The same procedure as for the violation of the constraint for the mean speed of the road service can be used. The eighth trial point in Table 5 is the point where the path of steepest descent meets this constraint. The modified direction of steepest descent changes into (11.1, 0, 8.625, 0, 18.05).

The path of steepest descent reaches a minimal point at trial 10. Both the mean speed of the road service and the mean speed of the sea service have reached their maximum value in this current local optimal point.

\section{Phase 2: Second order analysis}

Next, a new first order design is chosen with the optimum point recommended from the first analysis (trial 10 from Table 5) as central point. Since both the maximum of the main speed of the road service and the main speed of the sea service are reached, only three factors are incorporated in the new design. The factor levels for this design are calculated using the same range as in the initial design. These levels are given in Table 6. Again, five replications of the simulation are run for each experimental point.

Like before, the experimental points are standardized and, using regression analysis, a first order polynomial is fit to the specified points and their simulated results. This results in: 
Table 6 Levels for the three experimental factors

Factor name

Fixed order quantity $\left(X_{2}\right)$

Capacity road service $\left(X_{4}\right)$

Capacity sea service $\left(X_{6}\right)$
Lower

588

144

544

\begin{tabular}{lll}
\hline Factor name & Lower & Upper \\
\hline Fixed order quantity $\left(X_{2}\right)$ & 638 & 738 \\
Capacity road service $\left(X_{4}\right)$ & 156 & 182 \\
Capacity sea service $\left(X_{6}\right)$ & 569 & 619 \\
\hline
\end{tabular}

Table 7 Levels for the three experimental factors after decreasing the size of the region

Table 8 Levels for the axial points of the central composite design

\begin{tabular}{lll}
\hline Factor name & Lower & Upper \\
\hline Fixed order quantity $\left(X_{2}\right)$ & 604 & 772 \\
Capacity road service $\left(X_{4}\right)$ & 146 & 190 \\
Capacity sea service $\left(X_{6}\right)$ & 552 & 636 \\
\hline
\end{tabular}

$$
Y=474,120-841 x_{2}-289 x_{4}-9,954 x_{6},
$$

with $t$ test values (with 41 degrees of freedom) for the parameters under study: (313.263, $-0.524,-0.180,-6.201)$.

Values for the $R^{2}$-measure (0.448) and the $F$ test with degrees of freedom 3 and 41 (12.92) indicate that this regression does not explain much of the variation. Only the capacity of the sea service has a significant coefficient in the regression. Decreasing the step sizes and thereby reducing the size of the region of interest, can possibly make the region small enough to ensure that a first order approximation is an adequate local representation of the simulation response function. The factor levels for the design of the decreased experimental region are given in Table 7.

After having decreased the experimental region, a new regression analysis is performed. This results again in a regression where only the capacity of the sea service has a significant coefficient. Therefore, a second order polynomial is fit to these points.

In order to fit a second order polynomial to these points, the design needs to be adapted. It is possible to expand the first order design so that previously obtained simulation results can be re-used. One of the most commonly used second order designs is the central composite design (Wardrop and Myers 1990). The axial points that need to be simulated are $( \pm \alpha, 0,0),(0, \pm \alpha, 0)$ and $(0,0, \pm \alpha) . \alpha$ is often set to $N^{1 / 4}$, where $N$ is the number of factorial points, because this value guarantees a rotatable design (Myers et al. 1989). This gives us $\alpha=\left(2^{3}\right)^{(1 / 4)}$. The levels for $\alpha$ are given in Table 8 . 
Also five replications of the simulation are run at each of the additional experimental points. The second order polynomial is fitted as:

$$
Y=481,436-5,288 x_{2}+580 x_{2} x_{4}-454 x_{6}^{2}
$$

The $t$ test values (with 71 degrees of freedom of the coefficients) in the regression equation are: $(1707.226,-25.776,2.164,-2.035)$.

Both the $R^{2}(0.901)$ and the $F$ test value for overall significance of the regression (224.40) point out that the second order polynomial explains a large amount of the variation. Compared to the main effects only case, now linear, quadratic and interaction effects show significance. This response surface is also validated using the new data collection technique.

To find the minimizing point, the stationary points of the polynomial are determined using partial first derivatives. Because this minimizing point has to lie in the experimental region, constraints are imposed on the factors. The fixed order quantity has to lie between $-\alpha$ and $\alpha$, the capacity of the road service lies between $-\alpha$ and $\alpha$ and the capacity of the sea service lies in the interval $(-\alpha, \alpha)$. This leads to the following minimum: $(772,146,594)$. The total logistic costs at this point are 470,900.

\section{Conclusion}

The optimisation of a logistics network might include a lot of parameters influencing the total logistics cost. By setting up an experimental first-order design the important factors can be determined. Response surface methodology is used to determine an approximation of the optimal point in a multidimensional parameter space. Fine tuning towards the optimal point is done making use of a second-order design, based on the important factors, determined in the first phase. In such a way quadratic and interaction effects can be discovered in the space near the optimal point. After definition of the regression function and the estimation of its coefficients, the optimum can be determined.

\section{References}

Andradottir S (1995) A stochastic approximation algorithm with varying bounds. Opera Res 43(6):1037-1048

Andradottir S (1996) A scaled stochastic approximation algorithm. Manage Sci 42:475-498

Batmaz I, Tunali S (2003) Small response surface designs for metamodel estimation. Eur J Oper Res 145:455-470

Box GEP, Draper NR (1987) Empirical model-building and response surfaces. Wiley, New York

Dengiz B, Alabas C (2000) Simulation optimization using tabu search. In: Proceedings of the 2000 winter simulation conference, Orlando, 10-13 December 2000, pp 805-810

Goetschalckx M, Vidal CJ, Dogan K (2002) Modeling and design of global logistics systems: a review of integrated strategic and tactical models and design algorithms. Eur J Oper Res 143:1-18

Goldsman D, Kim S-H, Marshall WS, Nelson BL (2002) Ranking and selection for steady-state simulation: procedures and perspectives. INFORMS J Comput 14(1):2-19

Haddock J, Mittenthal J (1992) Simulation optimization using simulated annealing. Comput Ind Eng 22(4):387-395 
Ho Y-C, Cassandras CG, Chen C-H, Dai L (2000) Ordinal optimisation and simulation. J Oper Res Soc 51:490-500

Hodder JE, Dincer MC (1986) A multifactor model for international plant location and financing under uncertainty. Comput Oper Res 13(5):601-609

Hooke R, Jeeves TA (1961) A direct search solution of numerical and statistical problems. J Assoc Comput Mach 8:212-229

Jacobson SH, Schruben LW (1989) Techniques for simulation response optimisation. Oper Res Lett 8:1-9

Khuri AI, Cornell JA (1988) Response surfaces: designs and analyses. Marcel Dekker Inc, New York

Kleijnen JPC, Sargent RG (2000) A methodology for fitting and validating metamodels in simulation. Eur J Oper Res 120:14-29

Myers RH, Khuri AI, Carter WH Jr (1989) Response surface methodology: 1966-1988. Technometrics 31(2):137-157

Olafsson S, Kim J (2002) Simulation optimization. In: Proceedings of the 2002 winter simulation conference, San Diego, 8-11 December 2002, pp 79-84

Paul RJ, Chanev TS (1998) Simulation optimisation using a genetic algorithm. Simul Pract Theory 6:601-311

Pichitlamken J, Nelson BL (2001) Selection-of-the-best procedures for optimisation via simulation. In: Proceedings of the 2001 winter simulation conference, Arlington, 9-12 December 2001, pp 401-407

Ross PJ (1988) Taguchi techniques for quality engineering. McGraw-Hill Inc, New York

Safizadeh MH, Thornton BM (1984) Optimization in simulation experiments using response surface methodology. Comput Ind Eng 8(1):11-27

Vanmaele H, Van Landeghem R (1995) The integration of optimization paradigms and simulation practice in industrial management. Belg J Oper Res Stat Comput Sci 35(1):43-62

Vidal CJ, Goetschalckx M (1996) The role and limitations of quantitative techniques in the strategic design of global logistics systems. School of Industrial and Systems Engineering Research Report 96-023, Georgia Institute of Technology, Atlanta

Vidal CJ, Goetschalckx M (1997) Strategic production-distribution models: a critical review with emphasis on global supply chain models. Eur J Oper Res 98:1-18

Wardrop DM, Myers RH (1990) Some response surface designs for finding optimal conditions. J Stat Plan Inference 25:7-28 